\title{
Fungos conidiais associados a substratos vegetais submersos em algumas áreas do bioma Caatinga
}

Conidial fungi associated with submerged plant debris in some areas of Caatinga biome

\author{
Silvana Santos da Silva ${ }^{1,2}$, Tasciano dos Santos Santa Izabel ${ }^{1}$ \& Luís Fernando Pascholati Gusmão ${ }^{1}$
}

\begin{abstract}
Resumo
Os fungos conidiais desempenham importante papel em ecossistemas dulcícolas, sendo responsáveis pela decomposição de matéria orgânica. No bioma Caatinga, contudo, estudos abordando estes fungos ainda são incipientes. Neste estudo realizou-se um levantamento de fungos conidiais aquáticos associados a substratos vegetais submersos em ambientes lóticos, em um período de dois anos, em cinco áreas inseridas neste bioma (Brejo Paraibano-PB, APA da Chapada do Araripe e PARNA de Ubajara-CE, PARNA da Serra das ConfusõesPI, Serra da Jibóia-BA). Foram identificadas 90 espécies distribuídas em 62 gêneros. Duas foram classificadas como ingoldianas, duas como aero-aquáticas, e 86 como aquático-facultativas. A área que apresentou o maior número de espécies foi o Brejo Paraibano com 34 espécies, e com o menor número foi a APA da Chapada do Araripe com 20. Quanto aos substratos vegetais, a lâmina foliar apresentou o maior número de espécies, com 63. A similaridade entre as áreas, segundo índice de Sørensen, foi considerada baixa, com valores inferiores a 50\%. Os dados contribuem para o conhecimento da biodiversidade de fungos aquáticos no bioma Caatinga. Palavras-chave: ambiente lótico, fungos tropicais, biodiversidade, hifomicetos.
\end{abstract}

\begin{abstract}
The conidial fungi play an important role in freshwater ecosystems, being responsible for the decomposition of organic matter. In the Caatinga biome, however, studies addressing these fungi are still incipient. This study conducted a survey of aquatic conidial fungi associated with submerged plant debris in lotic environment, in a period of two years, in five areas included in this biome (BrejoParaibano-PB, APA da Chapada do Araripe e PARNA de Ubajara-CE, PARNA da Serra das Confusões-PI, Serra da Jibóia-BA). Ninety species were identified, distributed in 62 genera. Two species were classified as ingoldian fungi, two as aero-aquatic fungi, and 86 as aquatic-facultative fungi. The area of greatest number of species was Brejo Paraibano with 34 species, and the lowest number was of the APA da Chapada do Araripe with 20. In relation to a plant substrate, the leaf blade presented higher number of species, with 63 species. The similarity between the areas, according to Sørensen index, was considered low, with values less than $50 \%$. The data contribute to the knowledge of the biodiversity of aquatic fungi in Caatinga Biome.
\end{abstract}

Key words: biodiversity, lotic environment, hyphomycetes, tropical fungi.

\section{Introdução}

O bioma Caatinga, totalmente inserido no semiárido, é um bioma exclusivamente brasileiro. Caracteriza-se pelo clima quente, com menos de $1.000 \mathrm{~mm}$ de chuva por ano, distribuídos quase que completamente em períodos de três a seis meses. Devido a grande variação de chuva por ano, a vegetação neste bioma está submetida a uma deficiência hídrica sazonal, que ainda pode ser agravada por períodos de secas (Velloso et al. 2002). Este bioma apresenta uma grande variedade de tipos de vegetação: Matas Úmidas, Matas Estacionais, Cerrados, Tabuleiros, Campos Rupestres e remanescentes de Mata Atlântica (Velloso et al. 2002; Giulietti et al. 2006).

Velloso et al. (2002) indicaram nove enclaves de Mata Atlântica inseridos no bioma Caatinga: Serra da Ibiapaba/ Ubajara, Sobral, Itapajé, Serra do Baturité e Crato, no estado do Ceará; Brejo Paraibano e Camalaú, na Paraíba; Brejo da Madre

\footnotetext{
${ }^{1}$ Universidade Estadual de Feira de Santana, Av. Transnordestina s/n, C.P. 252 e 294, 44036-900, Feira de Santana, BA, Brasil

${ }^{2}$ Autor para correspondência: silvanasdasilva@hotmail.com
} 
de Deus em Pernambuco e Serra da Jibóia, na Bahia. Esses enclaves também são denominados de Brejos de Altitude, que, por estarem dentro do bioma Caatinga, também sofrem influência do clima semiárido, podendo ainda apresentar transições com as caatingas arbóreas, mata seca e as matas de cipó (Velloso et al. 2002; Thomas \& Britton 2008).

A grande quantidade de matéria orgânica fornecida por essas vegetações (folhas, cascas, galhos, flores e frutos) podem ser levadas para os ambientes aquáticos através de chuvas, ventos, escoamentos de águas superficiais e assoreamentos (Wong et al. 1998). Esse material é denominado alóctone e é de extrema relevância para a vida dos organismos aquáticos, onde os fungos, através da colonização e decomposição, os transformam em importante fonte de energia e de nutrientes para os sistemas dulcícolas (Vannote et al. 1980; Barlocher 2009).

Em uma revisão abordando a biodiversidade de fungos de ambientes de água doce, Goh \& Hyde (1996) consideraram a classificação para fungos conidiais aquáticos em quatro grupos ecológicos: os fungos ingoldianos, os fungos aero-aquáticos, os fungos terrestre-aquáticos e os fungos aquáticofacultativos. As definições destes grupos são baseadas nos aspectos morfológicos e no modo de vida destes organismos.

Os fungos ingoldianos dependem do ambiente aquático para a reprodução. Possuem conídios com formas hidrodinâmicas (tetrarradiados, sigmoides) que auxiliam a aderência ao substrato e também facilitam a dispersão. São encontrados crescendo e esporulando sobre substratos foliicolas e, em menor número, sobre substratos lignícolas submersos, ocorrendo geralmente em ambientes lóticos, que são caracterizados pelo fluxo contínuo de água (Goh \& Hyde 1996; Goh 1997). Entretanto, também podem ser encontrados associados a substratos vegetais submersos em ambientes lênticos, cujo fluxo de água é lento ou mesmo estagnado (Schoenlein-Crusius et al. 2009). Além destes substratos, os conídios são encontrados com facilidade nas espumas formadas em riachos.

Os fungos aero-aquáticos, grupo proposto por Beverwijk (1951), apresentam a capacidade de sobreviver vegetativamente associados a substratos submersos, contudo, esporulam apenas quando expostos ao ar (Goh \& Hyde 1996). Apresentam conídios com morfologia helicoidal ou clatróide, as quais permitem a retenção de ar para flutuação (Thomas 1996), e são mais comuns em ambientes lênticos, pois dependem das secas periódicas de seus habitats (Shearer et al. 2007).

Os fungos terrestre-aquáticos são encontrados em gotas de chuva ou orvalho acumuladas em partes de plantas, como a superfície foliar, ou espaços nos troncos, formando um filme d'água, onde são dispersos os conídios (Ando 1992). Os conídios produzidos são hialinos e, em menor número, dematiáceos e estaurosporos (Goh \& Hyde 1996).

Os fungos aquático-facultativos, termo proposto por Ingold (1975), tem como sinônimos: fungos aquáticos-submersos, sugerido por Goh \& Hyde (1996) e fungos aquáticos lignícolas (Goh 1997). Apresentam a capacidade de esporular e dispersar seus conídios nos ambientes terrestre e aquático, podendo se desenvolver tanto sobre substratos vegetais submersos quanto terrestres. São caracterizados como sapróbios e apresentam conidióforos e conídios com parede relativamente espessa (Goh \& Hyde 1996).

No Brasil estudos de fungos aquáticos se iniciaram na região Sudeste, no final da década de 1980 (Schoenlein-Crusius \& Milanez 1989). Outros trabalhos os sucederam: Schoenlein-Crusius \& Milanez 1998; Schoenlein-Crusius 2002; Schoenlein-Crusius et al. 2009), com foco para os fungos ingoldianos de regiões de Mata Atlântica e Cerrado, contudo, nesses trabalhos também são mencionados fungos aquático-facultativos

Schoenlein-Crusius \& Grandi (2003) em uma compilação de dados de literatura, reportaram 90 espécies de hifomicetos aquáticos para a América do Sul, através de investigações de ambientes lóticos e lênticos. Dentre os países investigados, o Brasil apresentou o maior número de fungos aquáticos, 59 espécies. Schoenlein-Crusius et al. (2009), em uma investigação de serapilheira mista submersa no Parque Estadual das Fontes do Ipiranga, um remanescente de Mata Atlântica no estado de São Paulo, identificaram 24 espécies de hifomicetos aquáticos. Em ambos os estudos houve predomínio de fungos ingoldianos.

No bioma Caatinga foram realizados alguns trabalhos com hifomicetos aquáticos: Barbosa et al. (2011), apresentaram uma nova espécie de hifomiceto aquático, Thozetella submersa F.R. Barbosa \& Gusmão; Barbosa \& Gusmão (2011), registraram 43 espécies de fungos conidiais de água doce, sendo sete novos registros para o ocidente, dois para o Neotrópico, nove para 
América do Sul, nove para o Brasil e dez para a Bahia; Almeida et al. (2012) registraram 17 espécies, dentre estas, cinco são novos registros (um para o continente americano, um para o Neotrópico, dois para a América do Sul e um para o Brasil); Fiuza \& Gusmão (2013) descreveram e ilustraram todas as espécies de Campylospora Ranzoni. Estes estudos investigaram substratos vegetais submersos em ambientes lóticos com enfoque para os fungos aquático-facultativos e ingoldianos, e apesar de serem ainda pontuais, já demonstram o grande número de espécies deste grupo para o bioma.

O presente estudo teve como objetivo realizar um levantamento taxonômico de fungos conidiais associados a substratos vegetais submersos, em ambientes lóticos de diferentes áreas inseridas no bioma Caatinga, e analisar a similaridade das comunidades fúngicas entre as áreas estudadas.

\section{Materiais e Métodos}

Áreas de estudo

Foram realizadas expedições de coleta em cinco áreas inseridas no bioma Caatinga (Tab. 1), sendo quatro delas enclaves de Mata Atlântica: Brejo Paraibano-(Paraíba), Área de Proteção Ambiental (APA) da Chapada do Araripe (Ceará), Parque Nacional (PARNA) de Ubajara (Ceará) e Serra da Jibóia (Bahia). E, uma área de Caatinga e ecótonos Cerrado/Caatinga: Parque Nacional (PARNA) da Serra das Confusões (Velloso et al. 2002).

Estas áreas foram avaliadas como prioritárias para conservação da biodiversidade (Velloso et al. 2002) e utilizadas pelo Programa de Pesquisa em Biodiversidade do Semiárido (PPBio/Semiárido).
Método de amostragem

Amostras de materiais vegetais em decomposição (folhas - sendo aqui divididas em lâmina foliar e pecíolo, cascas e galhos) submersos em rios e riachos foram coletadas nas diferentes áreas, e transportadas em sacos plásticos para o Laboratório de Micologia/Universidade Estadual de Feira de Santana (UEFS), onde passaram pelo procedimento de lavagem em água corrente durante 30 minutos. Em seguida, as amostras foram incubadas em câmara-úmidas (placas de Petri + papel filtro umedecido), e estas foram acondicionadas dentro de uma caixa de isopor (170 L) cujas paredes e tampa foram recobertas por papel toalha umedecido. Para manutenção da umidade, foi adicionado $500 \mathrm{ml}$ de água $+2 \mathrm{ml}$ de glicerina no fundo da caixa de isopor, que foi periodicamente aberta por cerca de 15 minutos para circulação do ar (modificado de Castañeda Ruiz 2005).

O material foi analisado sob estereomicroscópio Leica EZ4 durante 30 dias, para verificação da presença de estruturas reprodutivas (conidióforos, células conidiogênicas e conídios). Estas estruturas foram transferidas com auxílio de agulha tipo insulina $(12,7 \times 0,33 \mathrm{~mm})$ para lâminas contendo resina PVL (álcool polivinílico + ácido lático + fenol) (Trappe $\&$ Schenck 1982). Posteriormente, as lâminas foram depositadas na coleção do Herbário da Universidade Estadual de Feira de Santana (HUEFS).

A análise de similaridade das comunidades de fungos aquáticos entre as diferentes áreas foi realizada no programa PAST (Paleontological Statistics), utilizando-se o índice de similaridade de Sørensen e o algoritmo UPGMA. Uma matriz de similaridade foi feita baseada nos dados de presença (1) ou ausência (0) das espécies, para os cinco locais (Magurran 1988).

Tabela 1 - Aspectos gerais das áreas de estudo e período de coleta.

Table 1 - General aspect of study and collection period.

\begin{tabular}{|c|c|c|c|c|}
\hline Áreas de Estudo & Estado & Municípios coletados & Altitude (m) & Mês/ano de coleta \\
\hline APA da Chapada do Araripe & Ceará & $\begin{array}{c}\text { Santana do Cariri }\left(7^{\circ} 11^{\prime} 18^{\prime \prime} \text { S e } 39^{\circ} 44^{\prime} 13^{\prime \prime} \mathrm{O}\right) \text { e } \\
\text { Barbalha }\left(7^{\circ} 18^{\prime} 40^{\prime \prime} \mathrm{S} \text { e } 39^{\circ} 18^{\prime} 15^{\prime \prime} \mathrm{O}\right)\end{array}$ & 750 a 950 & Janeiro/ 2011 \\
\hline $\begin{array}{l}\text { PARNA da Serra das } \\
\text { Confusões }\end{array}$ & Piauí & Caracol $\left(09^{\circ} 16^{\prime} 43^{\prime \prime}\right.$ S e $\left.43^{\circ} 19^{\prime} 48^{\prime \prime} \mathrm{O}\right)$ & 700 & Abril/ 2011 \\
\hline Serra da Jibóia & Bahia & Santa Terezinha $\left(12^{\circ} 51^{\prime} \mathrm{S}\right.$ e $\left.39^{\circ} 28^{\prime} \mathrm{O}\right)$ & 750 a 800 & Junho/ 2011 \\
\hline Brejo Paraibano & Paraíba & $\begin{array}{l}\text { Areia }\left(6^{\circ} 58^{\prime} 12^{\prime \prime} \mathrm{S} \text { e } 35^{\circ} 42^{\prime} 15^{\prime \prime} \mathrm{O}\right) \\
\text { e Alagoa Nova }\left(7^{\circ} 40^{\prime} \mathrm{S} \text { e } 35^{\circ} 47^{\prime} \mathrm{O}\right)\end{array}$ & 420 a 618 & Novembro/ 2011 \\
\hline PARNA de Ubajara & Ceará & Ubajara $\left(3^{\circ} 51^{\prime} 16^{\prime \prime S}\right.$ e $\left.40^{\circ} 55^{\prime} 16^{\prime \prime O}\right)$ & 870 & Maio/2012 \\
\hline
\end{tabular}




\section{Resultados e Discussão}

A partir da análise das amostras coletadas em ambientes lóticos nas diferentes áreas, foram identificadas 90 espécies de fungos conidiais aquáticos (Tab. 2), pertencentes a 62 gêneros. Destes, 89 são hifomicetos e apenas um coelomiceto (Satchmopsis brasiliensis B. Sutton \& Hodges). O predomínio de hifomicetos em relação aos coelomicetos é comum em trabalhos com ambientes aquáticos (Sivichai et al. 2000; Cai et al. 2006a; Schoenlein-Crusius et al. 2009). Apenas treze espécies de coelomicetos foram registradas em ambiente de água doce (Shearer \& Raja 2013), e até o momento não se sabe quais fatores contribuem para essa baixa ocorrência. Segundo Descals \& Moralejo (2001), os coelomicetos são abundantes nestes ambientes, porém, muitas vezes são ignorados devido à dificuldade de identificar as espécies deste grupo. Shearer et al. (2007) acreditam que esse baixo número de espécies descritas para o ambiente aquático pode ser devido a falta de pesquisadores especializados no grupo.

Segundo a classificação de fungos conidiais de ambiente de água doce apresentada por Goh \& Hyde (1996), dentre as 90 espécies encontradas neste estudo, 86 são classificadas como fungos aquático-facultativos, apenas duas como fungos ingoldianos: Ingoldiella hamata D.E. Shaw e Triscelophorus acuminatus Nawawi; e duas como fungos aero-aquáticos: Inesiosporium longispirale (R.F. Castañeda) R.F. Castañeda \& W. Gams e Helicomyces roseus Link. Esses dados podem ser explicados pela metodologia utilizada que não favorece o aparecimento de fungos ingoldianos, uma vez que estes necessitam estar submersos para a esporulação, favorecendo assim, os fungos aquático-facultativos, que esporulam em contato com o ar (Goh \& Hyde 1996).

As espécies de fungos ingoldianos, I. hamata e T. acuminatus são muito comuns nos trópicos (Ingold 1975). Neste estudo, I. hamata se destaca por ser único anamorfo de um basiomiceto, evidenciado pela presença de ansas nos septos dos seus conídios. As demais espécies (Tab. 2) representam a fase assexual de ascomicetos.

Todas as espécies aqui encontradas já foram registradas associadas à serapilheira terrestre (Hughes \& Hennebert 1963; Ellis 1971; Castañeda Ruiz \& Kendrick 1990; Heredia-Abarca 1994; Wu \& Zhang 2005; Grandi \& Silva 2006; Marques et al. 2008; Barbosa et al. 2009; Castañeda Ruiz et al. 2010; Almeida et al. 2011; Santa Izabel et al. 2011, Seifert et al. 2011). Essa semelhança de espécies entre os ambientes aquáticos e terrestres pode ser explicada pela capacidade adaptativa destes fungos de sobreviverem nestes ambientes (Goh \& Hyde 1996), além da técnica de isolamento utilizada que favorece a coleta de fungos terrestres.

Dentre os gêneros encontrados, os mais representativos foram: Dictyochaeta Speg. e Ellisembia Subram. com cinco espécies cada, seguido de Xylomyces Goos, R.D. Brooks \& Lamore com quatro espécies. Esses gêneros são comumente encontrados em ambientes aquáticos (Goh \& Hyde 1999; Cai et al. 2003; Fryar et al. 2004). Os demais gêneros (Tab. 2) também já foram registrados nestes ambientes (Cai et al. 2006b).

Analisando o número de espécies por área (Fig. 1), o Brejo Paraibano apresentou o maior número, com 34 espécies, seguido do PARNA de Ubajara com 32, Serra da Jibóia com 31, PARNA da Serra das Confusões com 22 e APA da Chapada do Araripe com 20. Apenas duas espécies foram comuns a todas as áreas: Beltrania rhombica Penz. e Subulispora procurvata Tubaki. Ambas as espécies são comumente encontradas em ambiente aquático (Goh 1997; Smits et al. 2007) e terrestre (Heredia-Abarca 1994; Grandi \& Silva 2006; Marques et al. 2008; Barbosa et al. 2009; Santa Izabel et al. 2011), e são consideradas cosmopolitas devido a ampla distribuição geográfica (Grandi \& Gusmão 2002; Farr \& Rosman 2013). Em relação aos substratos, o maior número de espécies ocorreu associadas à lâmina foliar, com 63 espécies, e para os demais substratos, foram observadas 20 espécies

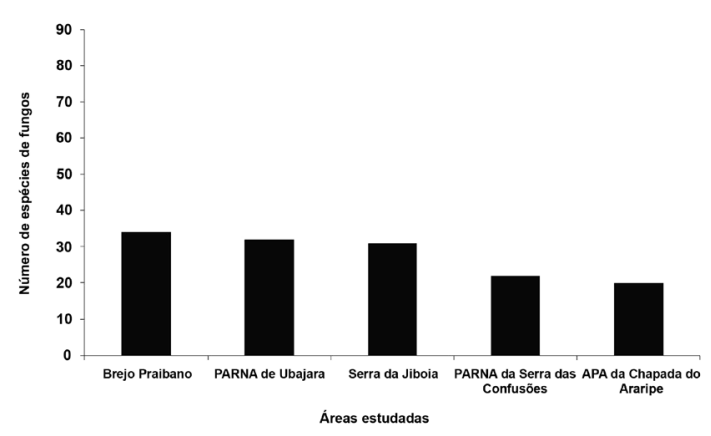

Figura 1 - Número de espécies de fungos conidiais associados a substratos vegetais submersos em cinco áreas inseridas no bioma Caatinga. Brejo Paraibano, PARNA de Ubajara, Serra da Jibóia-BA, PARNA da Serra das Confusões e APA da Chapada do Araripe.

Figure 1 - Number of species of conidial fungi associated with submerged plant debris in five areas included in the Caatinga biome. Brejo Paraibano, PARNA de Ubajara, Serra da Jibóia-BA, PARNA da Serra das Confusões e APA da Chapada do Araripe. 
Fungos conidiais e substratos vegetais submersos

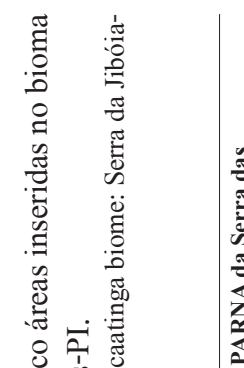

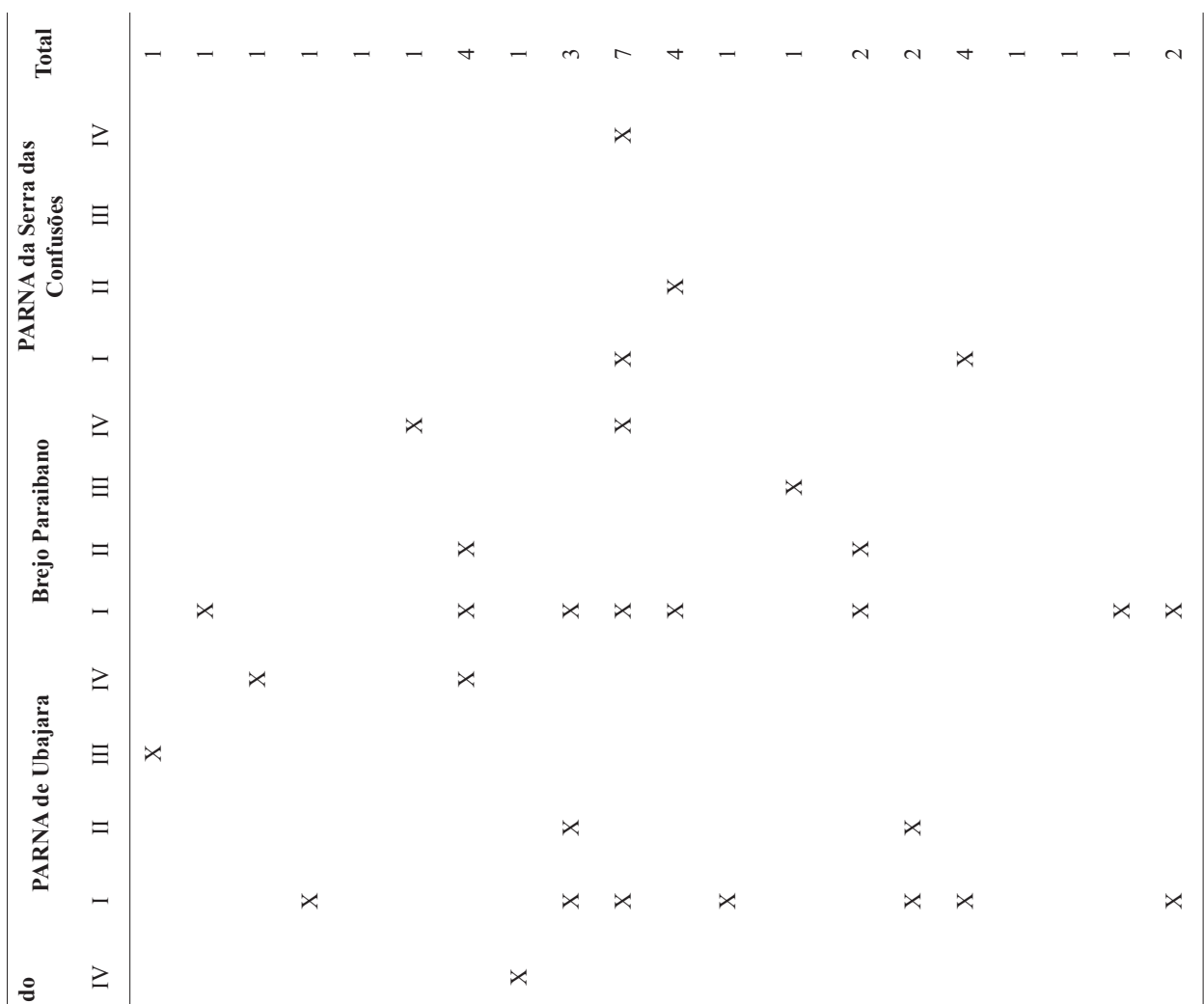

$0 \approx$

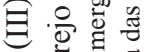

包产

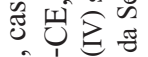

完.

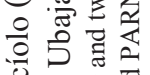

它 宁

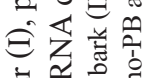

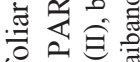

(1) 0

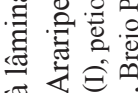

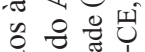

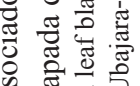

吾记

o

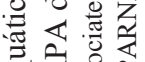

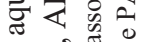

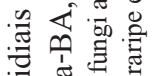

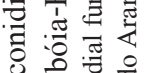

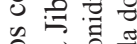

茄

可总

1 क ष

๙

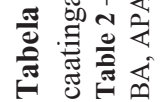
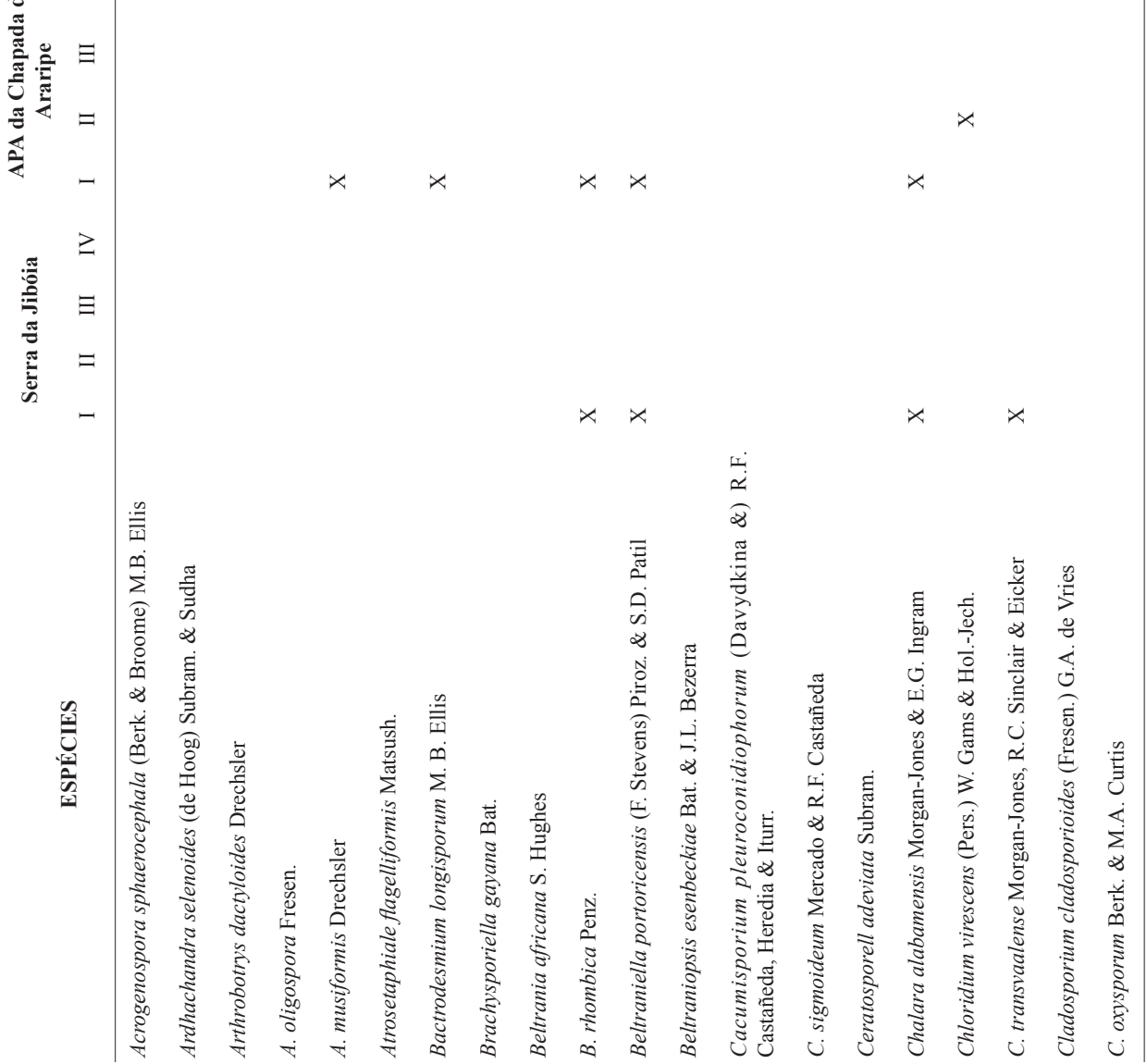

Rodriguésia 65(2): 527-538. 2014 


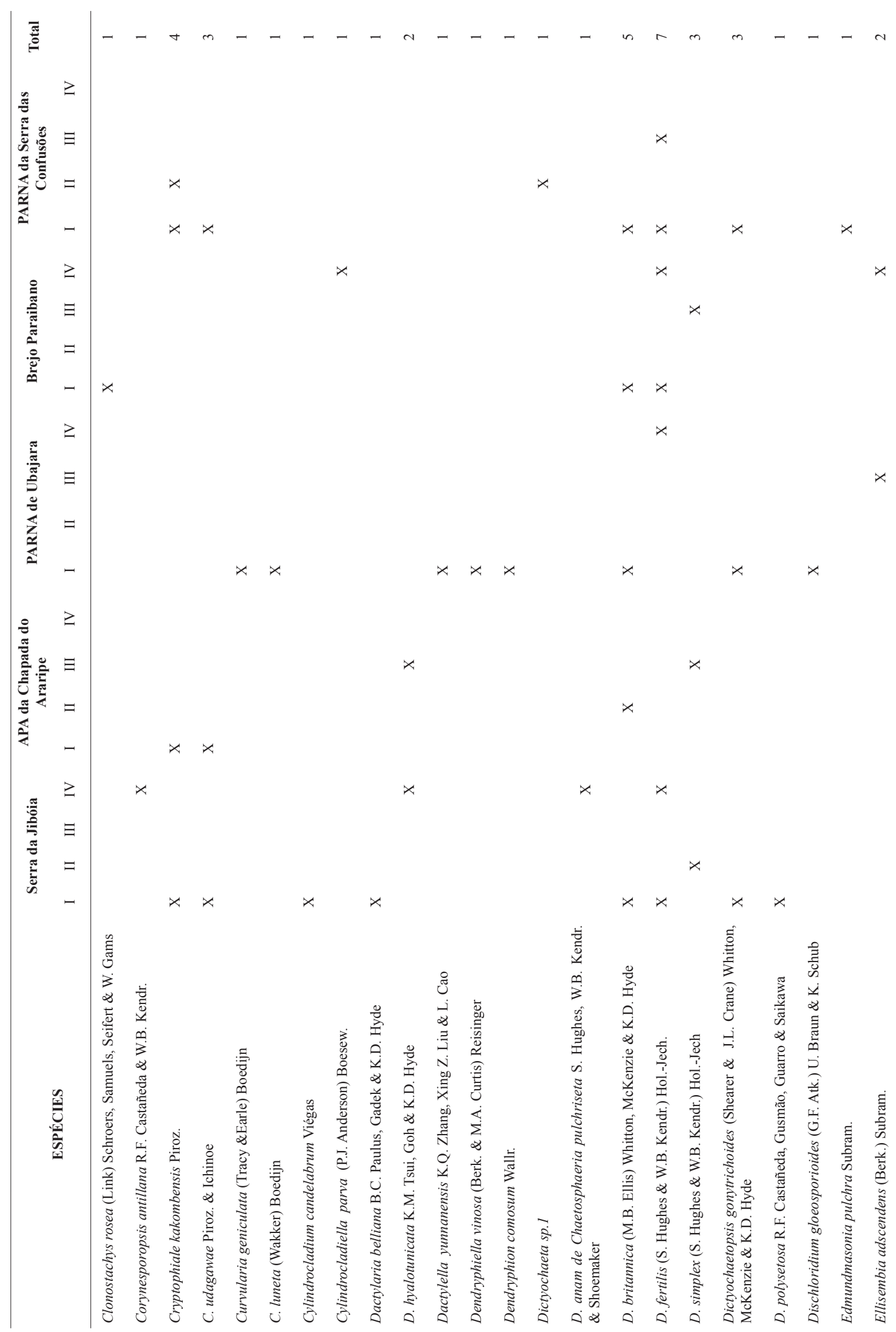


Fungos conidiais e substratos vegetais submersos

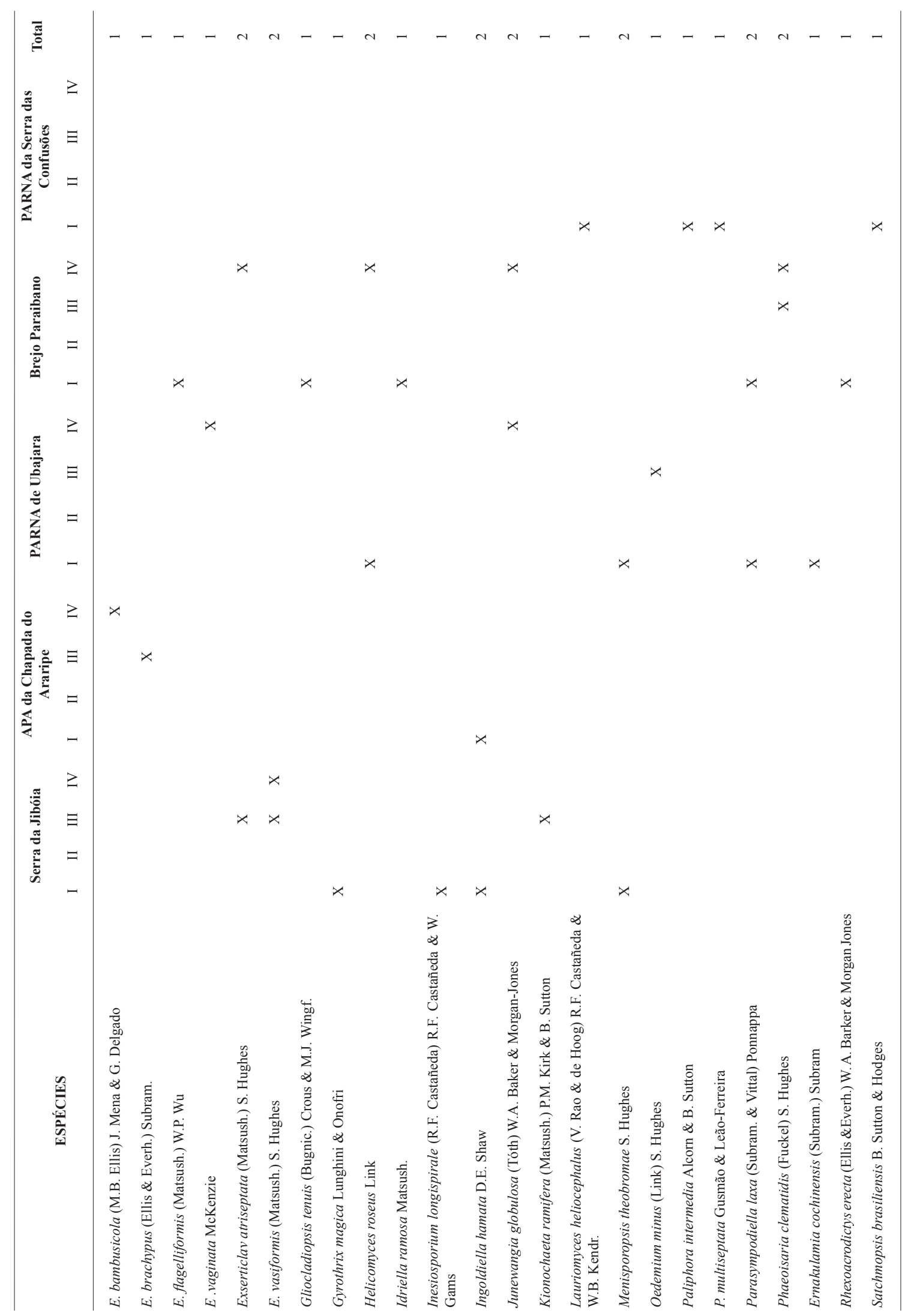

Rodriguésia 65(2): 527-538. 2014 


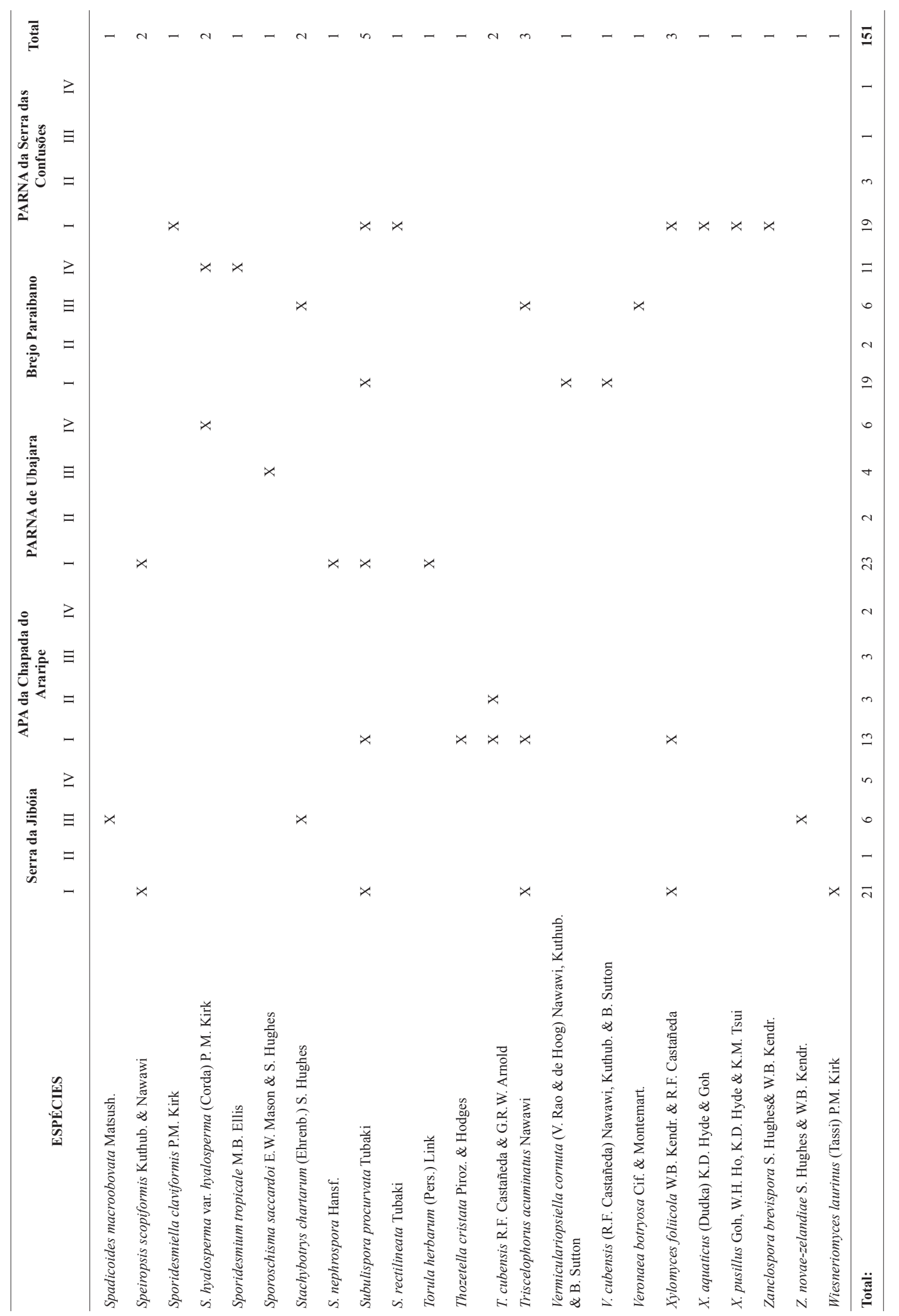


associadas a galho, 18 a casca e 11 a pecíolo (Fig. 2). Nenhuma espécie de fungo ocorreu em todos os substratos estudados.

A similaridade das comunidades de fungos entre as áreas foi considerada baixa (Tab. 3), apresentando valores abaixo de 0,5 . As maiores semelhanças foram observadas entre as áreas Serra da Jibóia e a APA da Chapada do Araripe (Índice de Sørensen=0,47), e entre Serra da Jibóia e o PARNA da Serra das Confusões (Índice de Sørensen=0,41), e a menor similaridade foi obtida entre o PARNA de Ubajara e APA da Chapada do Araripe (Índice de Sørensen $=0,15$ ). Esta baixa similaridade verificada entre as áreas, provavelmente pode ser explicada pela elevada diversidade de fungos existentes, o que torna mais difícil o isolamento de táxons similares através da técnica utilizada, além das diferenças na composição florística e fatores abióticos, entre estas áreas, o que também podem ter influenciado este resultado.

Marques et al. (2008) comparando comunidades de fungos entre duas áreas de Mata Atlântica obtiveram também uma baixa similaridade (Índice de Sørensen= 0,25), possivelmente pelas diferentes espécies vegetais e características ambientais. Hu et al. (2010) encontraram baixa similaridade (Índice de Sørensen= 0,12) de fungos entre os ambientes lótico e lêntico estudados, possivelmente devido a interferência do fluxo de água na comunidade de fungos aquáticos. Estes trabalhos demonstram a influência dos hospedeiros vegetais, e das características dos corpos d'água nas comunidades fúngicas.

A análise de agrupamento (Fig. 3) agrupou a Serra da Jibóia junto à APA da Chapada do Araripe e apresentou como grupo irmão o PARNA da Serra das Confusões, e o Brejo Paraibano agrupou com o PARNA de Ubajara. Esses agrupamentos podem ser justificados pelo período em que as coletas foram realizadas. Na Serra da Jibóia, na APA da Chapada do Araripe e no PARNA da Serra das Confusões, os riachos apresentaram baixo nível de água, e poucos substratos vegetais submersos disponíveis. Enquanto que, durante as coletas realizadas no Brejo Paraibano e no PARNA de Ubajara, os riachos e rios apresentaram alto fluxo de água e abundante quantidade de substratos vegetais.

O PARNA da Serra das Confusões apresenta transição entre fisionomias vegetais de Cerrado e Caatinga, enquanto as demais áreas caracterizamse por remanescentes de Mata Atlântica. Porém, todas as regiões estudadas compreendem o bioma

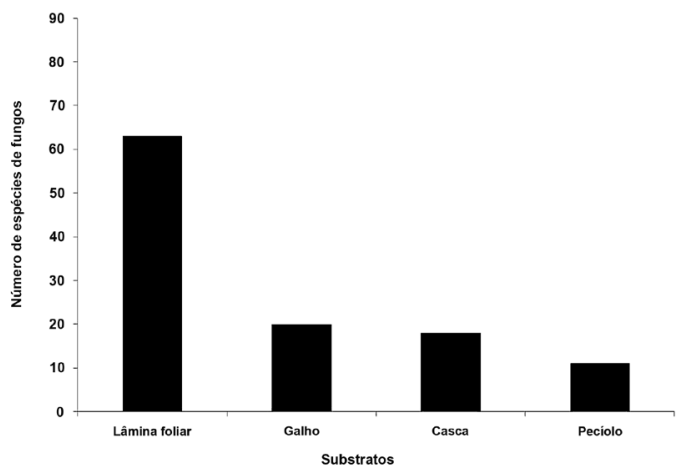

Figura 2 - Número de espécies de fungos conidiais encontrados em ambientes aquáticos, por substrato: lâmina foliar, galho, casca e pecíolo.

Figure 2 - Number of species of conidial fungi found in aquatic environment, for substrate: leaf blade, twig, bark and petiole.

Tabela 3 - Matriz de similaridade fúngica (Índice de Sørensen) entre as cinco áreas consideradas*

Table 3 - Similarity matrix fungal (Index Sørensen) among the five areas considered*

\begin{tabular}{cccccc}
\hline & A & B & C & D & E \\
\hline A & 1 & & & & \\
B & 0,4705 & 1 & & & \\
C & 0,2222 & 0,1538 & 1 & & \\
D & 0,3076 & 0,2963 & 0,3333 & 1 & \\
E & 0,4150 & 0,3809 & 0,2222 & 0,2142 & 1 \\
\hline
\end{tabular}

*Em que: A=Serra da Jibóia, B= APA da Chapada do Araripe, $\mathrm{C}=$ PARNA de Ubajara, $D=$ Brejo Paraibano, $E=$ PARNA da Serra das Confusões.

*Were: A=Serra da Jibóia, B=APA da Chapada do Araripe, $\mathrm{C}=$ PARNA de Ubajara, $\mathrm{D}=$ Brejo Paraibano, E= PARNA da Serra das Confusões.

Caatinga, em região semiárida. Portanto, sofrem influências climáticas desse domínio, além de apresentar transições onde se interpenetram dois ou até três tipos de vegetação (Velloso et al. 2002; Thomas \& Britton 2008), o que possibilita o aparecimento de espécies de fungos comuns entre as áreas. Além disso, a presença de espécies cosmopolitas e generalistas, como Beltrania rhombica, podem contribuir para que haja similaridade, mesmo que baixa, de áreas distintas.

Os resultados demonstraram um grande número de espécies de fungos associados a substratos vegetais submersos no bioma Caatinga, com uma composição taxonômica muito distinta entre as áreas $\mathrm{e}$, consequentemente, uma baixa similaridade. A diversidade de fungos aquáticos neste bioma, ainda pouco estudada, é muito relevante, uma vez que estes microrganismos desempenham importante papel 


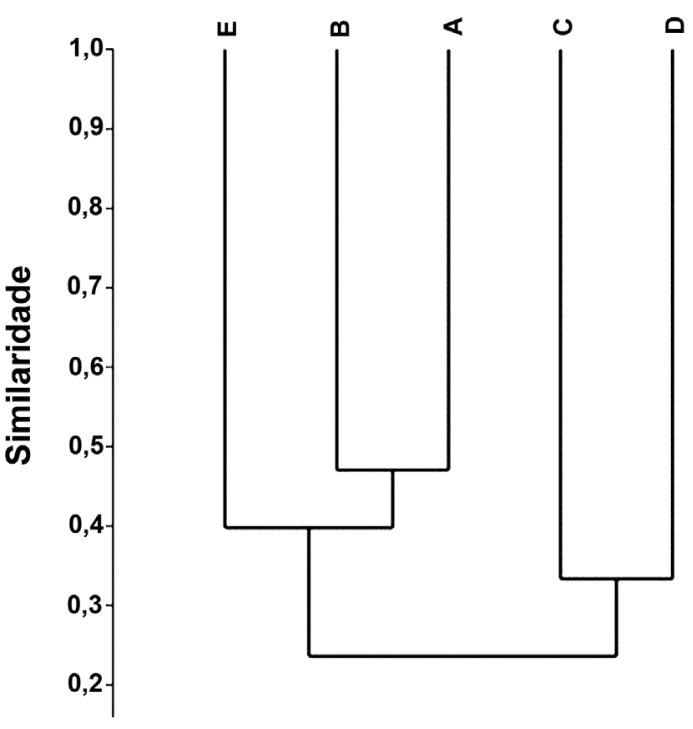

Figura 3 - Dendrograma gerado a partir do índice de similaridade de Sørensen e pelo método de agrupamento UPGMA entre as áreas consideradas. Em que: $A=$ Serra da Jibóia, $B=$ APA da Chapada do Araripe, $\mathrm{C}=\mathrm{PARNA}$ de Ubajara, $\mathrm{D}=$ Brejo Paraibano, $\mathrm{E}=$ PARNA da Serra das Confusões.

Figure 3-Dendrogram generated from the Sørensen similarity index and the UPGMA clustering method, among the areas considered. Where: $A=$ Serra da Jibóia, $B=A P A$ da Chapada do Araripe, $\mathrm{C}=$ PARNA de Ubajara, $\mathrm{D}=$ Brejo Paraibano, $\mathrm{E}=$ PARNA da Serra das Confusões.

no processo de decomposição do material alóctone submerso, contribuindo diretamente na cadeia alimentar nestes ecossistemas. Desta forma, estudos como o presente, permitem ampliar o conhecimento da diversidade deste grupo de fungos, fornecendo subsídios para futuros trabalhos de conservação.

\section{Agradecimentos}

Os autores agradecem ao Programa de Pósgraduação em Botânica-PPGBOT-UEFS. S.S. Silva e T.S. Santa Izabel agradecem ao Conselho Nacional de Desenvolvimento Científico e Tecnológico (CNPq) as bolsas concedidas de mestrado e doutorado, respectivamente. L.F.P. Gusmão agradece a bolsa produtividade (Proc. 303924/2008-0) concedida.

\section{Referências}

Almeida, A.C.A.; Barbosa, F.R. \& Gusmão, L.F.P. 2012. Alguns fungos conidiais aquático-facultativos do bioma Caatinga. Acta Botanica Brasilica 26: 918-926.
Almeida, D.A.C.; Santa Izabel, T.S. \& Gusmão, L.F.P. 2011. Fungos conidiais do bioma Caatinga I. Novos registros para o continente americano, Neotrópico, América do Sul e Brasil. Rodriguésia 62: 43-53.

Ando, K. 1992. A study of terrestrial aquatic hyphomycetes. Transactions of the Mycological Society of Japan 33: 415-425.

Barbosa, F.R. \& Gusmão, L.F.P. 2011. Conidial fungi from the semi-arid Caatinga biome of Brazil. Rare freshwater hyphomycetes and other new records. Mycosphere 2: 475-485.

Barbosa, F.R.; Silva, S.S.; Fiuza, P.O. \& Gusmão, L.F.P. 2011. Conidial fungi from the semi-arid Caatinga biome of Brazil. New species and records for Thozetella. Mycotaxon 115: 327-334.

Barbosa, F.R.; Maia L.C. \& Gusmão, L.F.P. 2009. Fungos conidiais associados ao folhedo de Clusia melchiorii Gleason e C. nemorosa G. Mey. (Clusiaceae) em fragmento de Mata Atlântica, BA, Brasil. Acta Botanica Brasilica 23: 79-84.

Barlocher, F. 2009. Reproduction and dispersal in aquatic hyphomycetes. Mycoscience 50: 3-8.

Beverwijk, van A.L. 1951. Zalewski's Clathrosphaera spirifera. Transactions of the British Mycological Society 34: 280-290.

Cai, L.; Zhang, K.; McKenzie, E.H.C. \& Hyde, K.D. 2003. Freshwater fungi from bamboo and wood submerged in the Liput River in the Philippines. Fungal Diversity 13: 1-12.

Cai, L.; Ji, K-F. \& Hyde, K.D. 2006a.Variation between freshwater and terrestrial fungal communities on decaying bamboo culms. Antonie van Leeuwenhoek 89: 293-301.

Cai, L.; Hyde, K.D. \& Tsui, C.K.M. 2006b. Genera of freshwater fungi. Fungal Diversity Researchs series 18. Fungal Diversity Press, Hong Kong. 261p.

Castañeda-Ruiz, R.F. 2005. Metodología en el estudio de los hongos anamorfos. In: Anais do V Congresso Latino Americano de Micologia, Brasília. Pp. 182-183.

Castañeda-Ruiz, R.F. \& Kendrick, B. 1990. Conidial fungi from Cuba II. University of Waterloo. Biology Series 33.

Castañeda Ruiz, R.F; Silveira-Simón, C.; Gené, J.; Guarro, J.; Minter, D.W.; Stadler, M \& Saikawa, M. 2010. A new species of Corynesporopsis from Portugal. Mycotaxon 114: 407-415.

Descals, E. \& Moralejo, E. 2001.Water and asexual reproduction in the ingoldian fungi. Botanica Complutensis 25: 13-71.

Ellis, M.B. 1971. Dematiaceous hyphomycetes. Common Wealth Mycological Institute, Kew. 608p.

Farr, D.F. \& Rossman, A.Y. 2013. Fungal Databases, Systematic Mycology and Microbiology Laboratory, ARS, USDA. Disponível em <http://nt.ars-grin.gov/ fungaldatabases/>. Acesso em 10 Jun 2013. 
Fiuza, P.O \& Gusmão, L.F.P. 2013. Ingoldian fungi from semiarid Caatinga biome of Brazil. The genus Campylospora. Mycosphere 4: 559-565.

Fryar, S.C.; Booth, W.; Davies, J.; Hodgkiss, I.J. \& Hyde, K.D. 2004. Distribution of fungion wood in the Tutong River, Brunei. Fungal Diversity 17: 17-38.

Giullieti, A.M.; Conceição, A. \& Queiroz, L.P. 2006. Diversidade e caracterização das fanerógamas do semi-árido brasileiro. Associação Plantas do Nordeste, Recife. 488 p.

Goh, T.K. 1997. Tropical freshwater hyphomycetes. In: Hyde, K.D. (ed.). Biodiversity of tropical microfungi. Hong Kong University Press, Hong Kong. Pp. 189-227.

Goh, T.K. \& Hyde, K.D. 1996. Biodiversity of freshwater fungi. Journal of Industrial Microbiology \& Biotechnology 17: 328-345.

Goh, T.K. \& Hyde, K.D. 1999. Fungi on submerged wood and bamboo in the Plover Cove Reservoir, Hong Kong. Fungal Diversity 3: 57-85.

Grandi, R.A.P. \& Gusmão, L.F.P. 2002. O gênero Subulispora Tubaki (fungos mitospóricosHyphomycetes) sobre folhas em decomposição no estado de São Paulo, Brasil. Hoehnea 29: 31-36.

Grandi, R.A.P. \& Silva, T.V. 2006. Fungos anamorfos decompositores do folhedo de Caesalpinia echinata Lam. Revista Brasileira de Botânica 29: 275-287.

Heredia-Abarca, G. 1994. Hifomicetes dematiaceos em Bosque Mesofilo de Montaña. Registros nuevos para Mexico. Acta Botanica Mexicana 27: 15-32.

Hu, D.M.; Cai, L.; Chen, H; Bahkali, A.H. \& Hyde, K.D. 2010. Fungal diversity on submerged wood in a tropical stream and an artificial lake. Biodiversity Conservation 19: 3799-3808.

Hughes, S.J. \& Hennebert, G.L. 1963. Microfungi X. Oedemium, Dimera, Diplosporium, Gongylocladium and Cladotrichum. Canadian Journal of Botany 41: 773-809.

Ingold, C.T. 1975. An illustrated guide to aquatic and waterborne hyphomycetes (fungi imperfect). Freshwater Biological Association Scientific Publication 30: 1-96.

Magurran, A.E. 1988. Ecological diversity and its measurement. Princeton University Press, Princeton. $177 \mathrm{p}$.

Marques, M.F.O.; Gusmão, L.F.P. \& Maia, L.C. 2008. Riqueza de espécies de fungos conidiais em duas áreas da Mata Atlântica no Morro da Pioneira, Serra da Jibóia, BA, Brasil. Acta Botanica Brasilica 22: 954-961.

Santa Izabel, T.S.; Santos, D.S.; Almeida, D.A.C. \& Gusmão, L.F.P. 2011. Fungos conidiais do bioma Caatinga II. Novos registros para o continente americano, Neotrópico, América do Sul e Brasil. Rodriguésia 62: 229-240.
Schoenlein-Crusius, I.H. 2002. Aquatic Hyphomycetes from cerrado regions in the state of São Paulo, Brazil. Mycotaxon 81: 457-462.

Schoenlein-Crusius, I.H. \& Grandi, R.A.P. 2003. The diversity of aquatic hyphomycetes in south America. Brazilian Journal of Microbiology 34: 183-193.

Schoenlein-Crusius, I.H. \& Milanez, A.I. 1989. Sucessão fúngica em folhas de Ficus microcarpa L.F., submersas no Lago Frontal situado no Parque Estadual das Fontes do Ipiranga, São Paulo. Revista de Microbiologia 20: 95-101.

Schoenlein-Crusius, I.H. \& Milanez, A.I. 1998. Fungal succession on leaves of Alchornea triplinervea (Spreng) M.Arg. Submerged in a stream of an Atlantic rainforest in the State of São Paulo, Brazil. Revista Brasileira de Botânica 21: 253-259.

Schoenlein-Crusius, I.H.; Moreira, C.G. \& Bicudo, D.C. 2009. Aquatic Hyphomycetes in the Parque Estadual das Fontes do Ipiranga - PEFI, São Paulo, Brazil. Revista Brasileira de Botânica 32: 411-426.

Seifert, K.; Morgan-Jones, G.; Gams, W. \& Kendrick, B. 2011. The genera of Hyphomycetes. CBS Biodiversity Series no. 9. CBS-KNAW Fungal Biodiversity Centre, Utrecht. 997p.

Shearer, C.A. \& Raja, H.A. 2013. Freshwate Ascomycetes Database. Disponível em $<$ http://fungi.life.illinois. edu>. Acesso em 12 Jan 2013.

Shearer, C.A.; Descals, E.; Kohlmeyer, B.; Kohlmeyer, J.; Marvanová, L.; Padgett, D.; Porter, D.; Raja, H.A.; Schmit, J.P.; Thorton, H.A. \&Voglymayr, H. 2007. Fungal biodiversity in aquatic habitats. Biodiversity Conservation 16: 49-67.

Sivichai, S.; Lones, E.B.G. \& Hywel-lones, N.L. 2000. Fungal colonisation of wood in a freshwater stream at KhaoYai National Park, Thailand. In: Hyde, K.D.; Ho, W.H. \& Pointing, S.B. (eds). Aquatic mycology across the millennium. Fungal Diversity 5: 71-88.

Smits, G.; Fernández, R. \& Cressa, C. 2007. Preliminary study of aquatic hyphomycetes from venezuelan streams. Estudio preliminar de hifomicetes acuáticos en cursos de agua dulce deVenezuela. Acta BotánicaVenezuelica 30: 345-355.

Thomas, K. 1996. Freshwater fungi. In: Grgurinovic (ed.). Fungi of Australia. Australian Biological Resources, Canberra. Pp. 1-37.

Thomas, W.W. \& Britton, E.G. (eds.). 2008. The Atlantic coastal forest of Northeastern Brazil. The New York Botanical Garden, New York. 586p.

Trappe, J.M. \& Schenck, N.C. 1982. Taxonomy of the fungi forming Endomycorrhizae. In: Schenck, N.C. (ed). Methods and principles of Mycorrhizae research. The American Phytopatological Society, Saint Paul. Pp. 1-9.

Vannote, R.L.; Minshall, W.G.; Cummins, K.W.; Sedell, J.R. \& Cushing, C.E. 1980. The river 
continuum concept. Canadian Journal of Fisheries and Aquatic Sciences 37: 130-37.

Velloso, A.L.; Sampaio, E.V.S.B. \& Pareyn, F.G.C. (eds). 2002. Ecorregiões: Propostas para o bioma Caatinga. Resultados do Seminário de Planejamento Ecorregional da Caatinga/Aldeia-PE. Associação Plantas do Nordeste, Instituto de Conservação Ambiental The Nature Conservancy do Brasil, Recife. 76p.
Wong, K.M.K.; Goh, T.K.; Hodgkiss, I.J.; Hyde, K.D.; Ranghoo, V.M.; Tsui, C.M.K.; Ho, W.H.; Wong, W.S. \& Yuen, T.C. 1998. The role of fungi in freshwater ecosystems. Biodiversity and Conservation 7: 1187-1206.

Wu, W. \& Zhang, W. 2005. Sporidesmium, Endophragmiella and related genera from China. Fungal Diversity Research Series 15. Hong Kong. Fungal Diversity Press. 351p. 